\title{
Endoscópio rígido e flexível na sinoscopia e técnica de triangulação em seios paranasais de equinos
}

\author{
Rigid and flexible endoscope in sinoscopy and triangulation technique in equine \\ paranasal sinus
}

Thaís Sodré de Lima Machado ${ }^{\mathrm{I}}$ Luís Cláudio Lopes Correia da Silva ${ }^{I I}$

RESUMO

A endoscopia dos seios paranasais, denominada sinoscopia, trata-se de técnica minimamente invasiva efetiva para diagnóstico e tratamento das afecções sinusais. Este trabalho teve como objetivo determinar portais para triangulação, a partir dos pontos de sinoscopia já descritos e comparar a utilização de endoscópio rígido e flexível de pequeno calibre durante a sinoscopia em equinos de diferentes idades. Foram realizados, sequencialmente três procedimentos: sinoscopia com endoscópio rígido, sinoscopia com endoscópio flexível e abordagem cirúrgica por técnica de triangulação. A qualidade da imagem obtida com o endoscópio rígido foi superior à do endoscópio flexível, entretanto, a amplitude de visão foi maior com o endoscópio flexível, que permitiu maior mobilidade e aproximação das estruturas anatômicas dos seios paranasais. A partir dos portais situados sobre o seio frontal, foi possível examinar tanto o seio frontal como o maxilar caudal. Através do portal do seio maxilar caudal, foram examinadas as raízes dentárias e demais estruturas localizadas no interior deste. O exame do seio maxilar rostral foi dificultado em alguns casos devido ao acentuado tamanho da raiz do $1^{\circ}$ dente molar, principalmente em animais jovens. Os portais para técnica de triangulação foram determinados nos seios frontal e maxilar caudal, permitindo acesso de instrumental cirúrgico às estruturas anatômicas. Através dos resultados obtidos foi possível estabelecer portais para realização de técnicas de sinoscopia e triangulação no diagnóstico e tratamento das afecções sinusais de equinos, utilizando endoscópio rígido ou flexível.

Palavras-chave: sinoscopia, equino, técnica triangulação.

\section{ABSTRACT}

Sinoscopy, the paranasal sinus endoscopy, is a minimally invasive technique used to diagnose and treat sinus disease. The objective of this study was to determine triangulation portals sites from sinoscopy portals previously described and to compare rigid and flexible endoscope of small diameter in horses

\begin{abstract}
of different ages. Paranasal sinuses underwent three procedures in sequence: sinoscopy with rigid endoscope, sinoscopy with flexible endoscope and surgical approach to triangulation technique. Rigid endoscope presented higher image quality, although the amplitude of visualization was greater with flexible endoscope that yielded better mobility and proximity of the paranasal sinus anatomical structures. Through frontal sinus portals, the frontal and caudal maxillary sinuses were examined. The structures of maxillary caudal sinus, including the roots of the cheek teeth, were observed through the portal of caudal maxillary sinus. In some animals, the bigger root of the first upper molar teeth, mainly in young horses, made the exam of rostral sinus exam harder. Triangulation portals were determined in frontal and caudal maxillary sinus enabling surgical instrumentation access to anatomical structures. The results enabled the establishment of sinoscopy and triangulation portals to diagnosis and treatment of sinus disease in horses using either flexible or rigid endoscope.
\end{abstract}

Key words: sinoscopy, equine, triangulation technique.

\section{INTRODUÇÃO}

O equino possui seis pares de seios paranasais: frontal, conchal dorsal, esfenopalatino, maxilar caudal, maxilar rostral e conchal ventral (HILLMANN, 1975). Os primeiros quatro compartimentos comunicam-se entre si, assim como o seio maxilar rostral e o conchal ventral, originando duas grandes cavidades sinusais funcionais. Recentemente, um novo par de seios paranasais, o etmoidal, foi descrito por O`LEARDY \& DIXON (2011). A drenagem dos seios frontal, conchal dorsal e etmoidal para o seio maxilar caudal ocorre através

IDepartamento de Clínica Médica, Faculdade de Medicina Veterinária e Zootecnia, Universidade de São Paulo (FMVZ-USP), São Paulo,
SP, Brasil. E-mail: tsodrelima@gmail.com. Autor para correspondência.

IIDepartamento de Cirurgia, FMVZ-USP, São Paulo, SP, Brasil. 
da abertura frontomaxilar (FREEMAN, 1991). Em cavalos jovens, o interior dos seios maxilar rostral e caudal é ocupado pelo ápice do quarto dente prémolar e do primeiro, segundo e terceiro dentes molares. À medida que ocorre a erupção desses dentes, o espaço interno dos seios maxilares aumenta (HILLMANN, 1975). Os seios maxilares rostral e caudal estão separados por um septo ósseo, localizado geralmente entre o primeiro e segundo dentes molares (FREEMAN, 1991). A comunicação sinonasal pode ocorrer através de um ou dois orifícios, constituindo uma estreita via de drenagem (1-2 mm) para o aspecto caudal do meato médio nasal (PROBST et al., 2005; O’LEARY; DIXON, 2011).

As afecções que acometem os seios paranasais possuem importância clínica, com destaque à marcante tendência a cronicidade e dificuldade para obtenção de diagnóstico definitivo, levando à baixa eficácia do tratamento. Entre as enfermidades mais frequentes na espécie equina, estão os casos de sinusite primária, sinusite secundária a distúrbios dentais, hematoma etmoidal, cistos e neoplasias (DIXON et al., 2012a).

Os meios auxiliares tradicionais para o diagnóstico dos distúrbios sinusais incluem a avaliação dos sinais clínicos, exame radiográfico, exame endoscópico das vias aéreas anteriores e aspiração do conteúdo sinusal para posterior exame citológico ou bacteriológico.

Mesmo sendo muito úteis, as informações obtidas por esses exames podem não ser totalmente esclarecedoras. A exploração cirúrgica pela técnica de sinusotomia pode levar ao diagnóstico definitivo, porém, além de ser procedimento invasivo, requer a manutenção do animal sob anestesia geral e apresenta maior risco de complicações no período pós-operatório (RUGGLES et al., 1991; BEARD \& HARDY, 2001). Por outro lado, por se tratar de procedimento minimamente invasivo, a sinoscopia pode ser realizada em cavalos sedados, mantidos em posição quadrupedal, permitindo o exame detalhado dos seios paranasais, realização de biópsias e coleta de material para cultura e antibiograma, sendo indicada principalmente nos casos com diagnóstico inconclusivo (RUGGLES et al.,1993; O’LEARY\& DIXON, 2011). Pequenas intervenções terapêuticas utilizando a sinoscopia para casos de sinusite primária, hemorragia, ou pequenas formações císticas já foram descritas (MARQUES et al., 2001; RUGGLES et al.,1993; SILVA et al., 2004; SILVA et al., 2009; DIXON \& O’LEARY, 2012; DIXON et al., 2012b). Entretanto, para que a sinoscopia seja utilizada com finalidade terapêutica, faz-se necessária, na maioria dos casos, a associação da técnica de triangulação (RUGGLES \& BERTONE, 1997).

O princípio da triangulação é um dos alicerces da cirurgia endoscópica e consiste na criação de portais adicionais que possibilitam que o endoscópio e os instrumentos de endoscopia sejam introduzidos de forma a conduzir a realização de biópsias ou procedimentos terapêuticos dentro de cavidades corpóreas. Os pontos ideais para a triangulação são obtidos quando ocorre a visualização bem sucedida dos instrumentos, através do endoscópio, e a área a ser manipulada permaneça no ápice do triângulo formado pela extremidade da óptica e do instrumental (WILLARD \& SCHULZ, 2008).

O objetivo do presente estudo foi determinar a localização de portais adicionais para a técnica de triangulação por Sinoscopia, ainda não descritos na literatura, e comparar a utilização do endoscópio rígido e flexível de pequeno calibre em equinos de diferentes idades, apontando as vantagens e desvantagens de cada instrumento.

\section{MATERIAIS E MÉTODOS}

Peças anatômicas: Foram utilizadas 24 peças anatômicas, correspondentes à cabeça de equinos submetidos à eutanásia por causas diversas, sem histórico de doença sinusal prévia, colhidas junto ao Serviço de Cirurgia de Grandes Animais da Faculdade de Medicina Veterinária e Zootecnia da Universidade de São Paulo - FMVZ-USP.

Para adequação eaprimoramento da técnica, utilizando os endoscópios rígido e flexível, bem como para determinação dos portais para triangulação, foram utilizadas quatro peças anatômicas. As demais foram divididas em quatro grupos, com cinco peças cada, de faixa etária, respectivamente, de 1,5 a 4 anos (GI), 5 a 8 anos (GII), 9 a 12 anos (GIII) e de 13 anos ou mais (GIV). Tal distribuição foi embasada no fato de que os seios paranasais são preenchidos pelas raízes dos dentes molares e pré-molares de forma decrescente, conforme a idade.

Técnica de Sinoscopia: O acesso às cavidades sinusais foi obtido com auxílio de furadeira e broca de 8,0mm de diâmetro. Quatro pontos para acesso aos seios paranasais foram determinados durante a fase de treinamento, embasados nos locais previamente descritos por RUGGLES et al. (1991), com pequenas modificações. Sobre o seio frontal, foram estabelecidos dois portais, localizados 3,0 e $5,0 \mathrm{~cm}$ lateralmente à linha média, ambos $0,5 \mathrm{~cm}$ caudal à linha que une os cantos mediais dos olhos (SF3 e SF5, respectivamente). O portal sobre o seio 
maxilar caudal (SMC) situou-se 3,0 cm rostral e 2,5cm ventral ao canto medial do olho. O local estabelecido para sinoscopia no seio maxilar rostral foi $1,5 \mathrm{~cm}$ dorsal e 2,0cm caudal, ao limite rostral da crista facial (SMR) (Figura 1). Para descrição e documentação das estruturas anatômicas examinadas, o endoscópio rígido com 4,0mm de diâmetro e $30^{\circ}$ de angulação foi introduzido pelos portais SF3, SF5, SMC e SMR nos lados direito e esquerdo de cada peça anatômica avaliada. Em seguida, o mesmo procedimento foi realizado com endoscópio flexível, com 4,8mm de diâmetro, com a finalidade de se comparar as estruturas observadas e a amplitude de visão obtida com os endoscópios.

Técnica de triangulação: A localização dos portais para triangulação foi determinada levandose em conta a facilidade de acesso do probe (gancho rombo para palpação indireta) às estruturas no interior dos seios paranasais, permitindo o alcance dessas.

Análise dos resultados: Os resultados estão apresentados considerando-se a idade dos animais e o tipo de endoscópio utilizado, levando-se em conta a qualidade de imagem; a amplitude de visão; as estruturas anatômicas observadas; o número e localização dos acessos para técnica de triangulação; e a possibilidade de inspeção em mais de um seio utilizando-se o mesmo portal (SF3, SF5, SMC ou SMR).

\section{RESULTADOS E DISCUSSÃO}

Equipamentos: à semelhança do exame com o endoscópio rígido, a utilização do endoscópio flexível de pequeno diâmetro (4,8mm) para realização de sinoscopia necessitou apenas de um portal com 8,0 mm de diâmetro para acesso aos seios paranasais. Esse fato torna-se uma vantagem quando comparamos ao portal de $14,0 \mathrm{~mm}$, necessário para realização de sinoscopia com endoscópio flexível de 9,0 ou 10,0 mm de diâmetro, relatado por WOSTER \& HACKETT (1999).

Apesar da manipulação do endoscópio flexível gerar maior grau de complexidade para o direcionamento de sua extremidade, a amplitude de visão foi superior, devido, em grande parte, à possibilidade da realização de manobras de flexão, atingindo pontos não acessíveis ao endoscópio rígido, como é o caso dos seios conchal ventral e esfenopalatino, através dos portais sobre o seio maxilar rostral e seio frontal, respectivamente. Esse fato foi comprovado ao analisar a progressão dos endoscópios em exame radiográfico, realizado durante os procedimentos (Figura 2 A e B). No seio esfenopalatino, foi possível introduzir até $7,0 \mathrm{~cm}$ desse endoscópio a partir do portal SF5, manobra inviável com a óptica rígida. Para o exame do seio conchal dorsal, o endoscópio flexível também apresentou maior progressão rostral.

As raízes dentárias localizadas no seio maxilar caudal foram inspecionadas com maior proximidade pelo endoscópio flexível, através dos portais do seio frontal. No seio maxilar rostral, esse endoscópio apresentou maior progressão, resultando em exame mais detalhado das estruturas. Nas peças anatômicas cuja raiz dentária preenchia praticamente

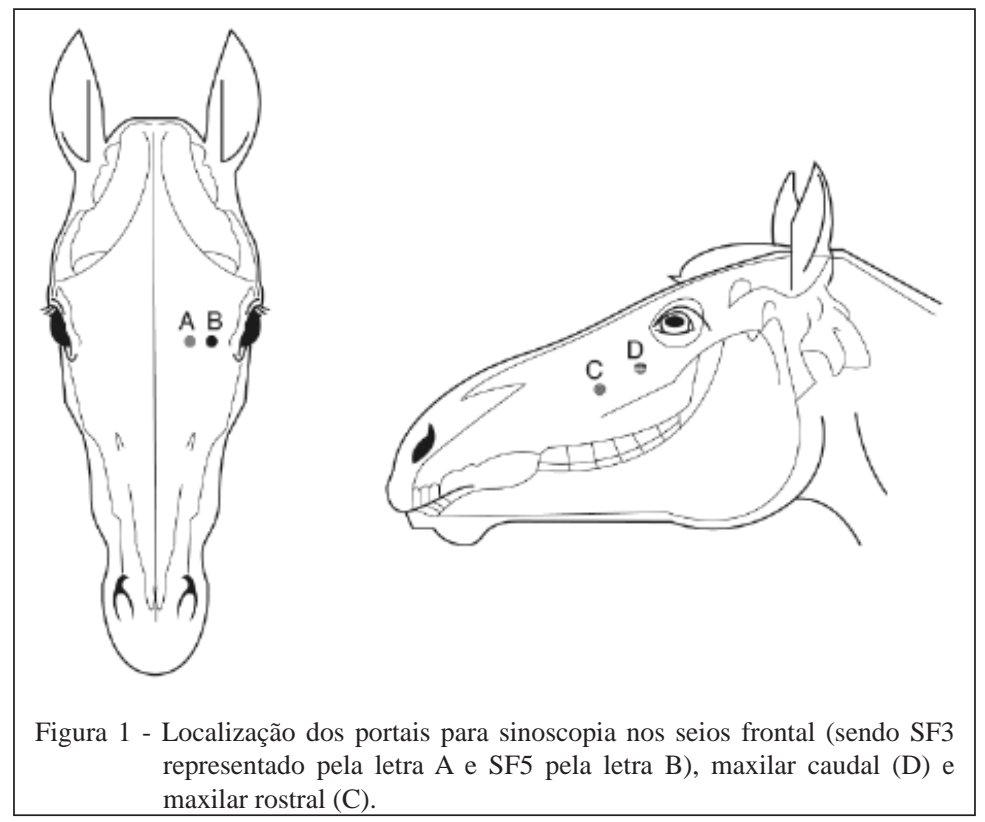

Ciência Rural, v.43, n.12, dez, 2013. 
a totalidade da cavidade sinusal, a amplitude de visão com o endoscópio rígido foi superior, em decorrência da angulação da lente $\left(30^{\circ}\right)$. Entretanto, a qualidade da imagem fornecida pelo endoscópio rígido foi superior, quando comparada com o endoscópio flexível, tanto pela definição como amplificação da imagem, facilitando a percepção de maiores detalhes (Figuras 2 C e D).

Portais de sinoscopia e estruturas anatômicas: durante a fase de treinamento em peças anatômicas, foram determinados quatro portais para sinoscopia, de acordo com a melhor qualidade de imagem obtida, identificação de maior número de estruturas anatômicas através de um único acesso e redução do risco de trauma às estruturas adjacentes, principalmente durante a utilização da furadeira.

Os locais estabelecidos para sinoscopia frontal visaram a otimização da visualização das estruturas dos seios paranasais frontal, conchal dorsal, etmoide, esfenopalatino e maxilar caudal por um único portal, bem como a redução de risco de traumatismo no etmoide ao se criar o portal, sendo os portais localizados $3,0 \mathrm{~cm}$ (SF3) e 5,0 cm (SF5) lateralmente à linha média, ambos $0,5 \mathrm{~cm}$ caudal à linha que une os cantos mediais dos olhos (Figura 1). O seio frontal, conchal dorsal, etmoidal e a abertura frontomaxilar foram identificados tanto pelo portal SF3, como pelo SF5, em todas as peças utilizadas, independente do grupo e do tipo de endoscópio empregado (Figura 3).

Através desses portais, foi possível passar ambos os endoscópios pela abertura frontomaxilar e observar as estruturas do seio maxilar caudal: canal infraorbitário, septo do seio maxilar, bulha conchal ventral e as raízes do $2^{\circ}$ e $3^{\circ}$ dentes molares (Figura 3). Entretanto, a maior proximidade do portal SF3 ao etmoide fez com que essa estrutura fosse atingida com a broca em três peças anatômicas e dificultou a passagem dos endoscópios pela abertura frontomaxilar. Por esse motivo, em alguns casos, foi possível observar maior número de estruturas anatômicas do seio maxilar caudal e o interior do seio esfenopalatino pelo SF5 do que pelo SF3, assim como constatado por RUGGLES et al. (1991), que recomendaram esse portal tanto para o exame do seio frontal, como do maxilar caudal. Outra vantagem dos portais SF3 e SF5 é a lavagem mais eficaz das cavidades sinusais caudais durante tratamento de sinusites, quando comparado ao portal SMC (DIXON \& O’LEARY, 2012).

A localização do acesso pelo seio maxilar caudal, diferente do proposto por RUGGLES et al. (1991), foi estipulada a $3,0 \mathrm{~cm}$ rostral e $2,5 \mathrm{~cm}$ ventral ao canto medial do olho (SMC) (Figura 1). Essa localização garantiu um exame mais aproximado das raízes por estar justamente entre as raízes dentárias dos $2^{\circ}$ e $3^{\circ}$ dentes molares, sendo extremamente útil quando da suspeita de envolvimento dentário na afecção sinusal. Por ser uma cavidade ampla, o tamanho das raízes dentárias nos animais jovens não interferiu na qualidade do exame do seio maxilar caudal.

Neste estudo, durante a sinoscopia pelo portal SMC, foram identificadas, em todas as peças utilizadas nos quatro grupos, a bulha conchal ventral, o canal infraorbitário, raízes dentárias do $2^{\circ}$ e $3^{\circ}$ dentes molares e septo do seio maxilar, localizados no seio maxilar caudal; e a abertura frontomaxilar e o etmoide no seio frontal. O interior do seio esfenopalatino não foi observado em nenhuma peça estudada através deste portal. Em uma peça anatômica do GII, o septo do seio maxilar apresentou-se entre o $2^{\circ}$ e $3^{\circ}$ dentes molares, tanto no lado direito como no esquerdo; e, em duas peças do GI, o septo do seio maxilar estava localizado entre o $4^{\circ}$ dente pré-molar e o $1^{\circ}$ molar.

WORSTER \& HACKETT (1999) utilizaram, para exame do seio maxilar caudal e seio esfenopalatino, o portal localizado $0,5 \mathrm{~cm}$ rostral e $1,0 \mathrm{~cm}$ ventral ao canto medial do olho. Tanto no presente estudo, como no realizado por RUGGLES et al. (1991), o exame do seio esfenopalatino foi realizado com sucesso a partir do portal localizado no seio frontal. Isso torna desnecessária a criação de um portal tão próximo ao globo ocular para o exame do seio esfenopalatino, principalmente em animais apenas sedados.

As estruturas anatômicas passíveis de observação endoscópica no seio maxilar rostral são a raiz do $1^{\circ}$ dente molar, septo maxilar, canal infra-orbitário e entrada para o seio conchal ventral. O local estabelecido para o portal SMR reduziu risco de lesão no músculo elevador nasolabial e a dificuldade em se realizar o exame. Mesmo assim, o tamanho do seio maxilar rostral mostrou-se reduzido, principalmente nas peças do GI e GII, onde as raízes dentárias protuberantes dificultaram ou até mesmo impossibilitaram o exame. Isso ocorreu em uma peça com o endoscópio rígido e em duas peças com o flexível no GI e em duas peças no GII, utilizando ambos os endoscópios. RUGGLES et al. (1991) também relataram dificuldade em examinar esse seio paranasal, devido ao tamanho da cavidade. Outra possível complicação da sinoscopia no seio maxilar rostral é a lesão da raiz do $1^{\circ}$ dente molar com a broca, ao criar o portal. Para reduzir a incidência de lesões envolvendo a raiz dentária, BARAKZAIET al. (2008) 


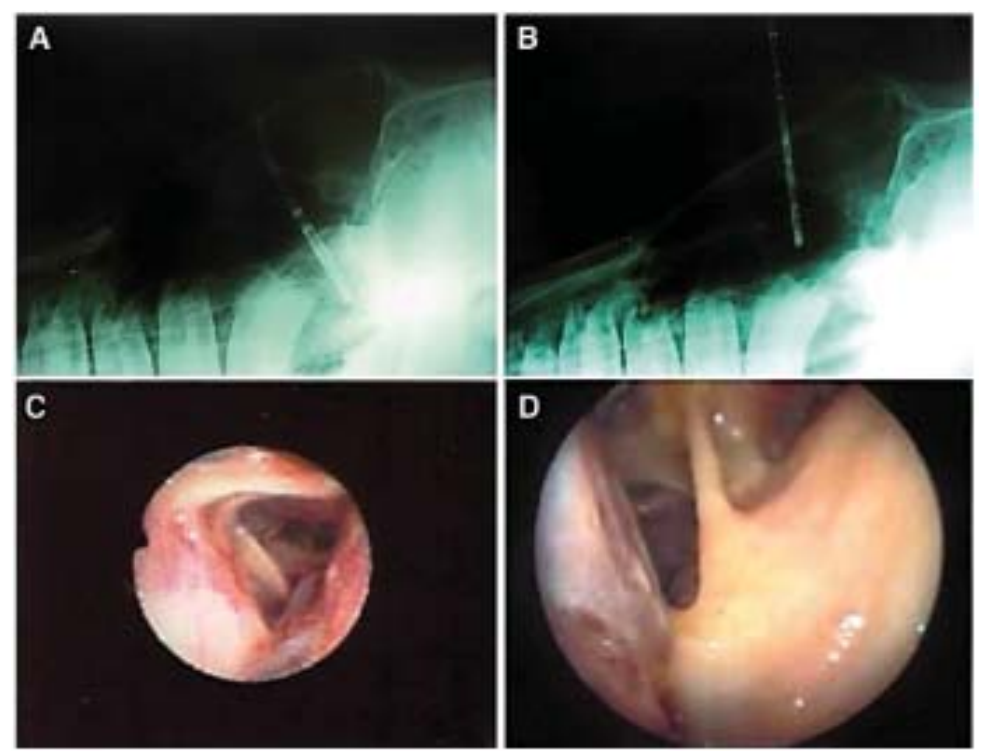

Figura 2 - Imagem radiográfica obtida durante sinoscopia a partir do portal SF5, sendo: A) endoscópio flexível, posicionado no interior do seio esfenopalatino; B) endoscópio rígido, posicionado na entrada do seio esfenopalatino. Imagem obtida a partir do SF5, sendo: A) endoscópio flexível, posicionado no interior do seio esfenopalatino; B) endoscópio rígido, posicionado na entrada do seio esfenopalatino.

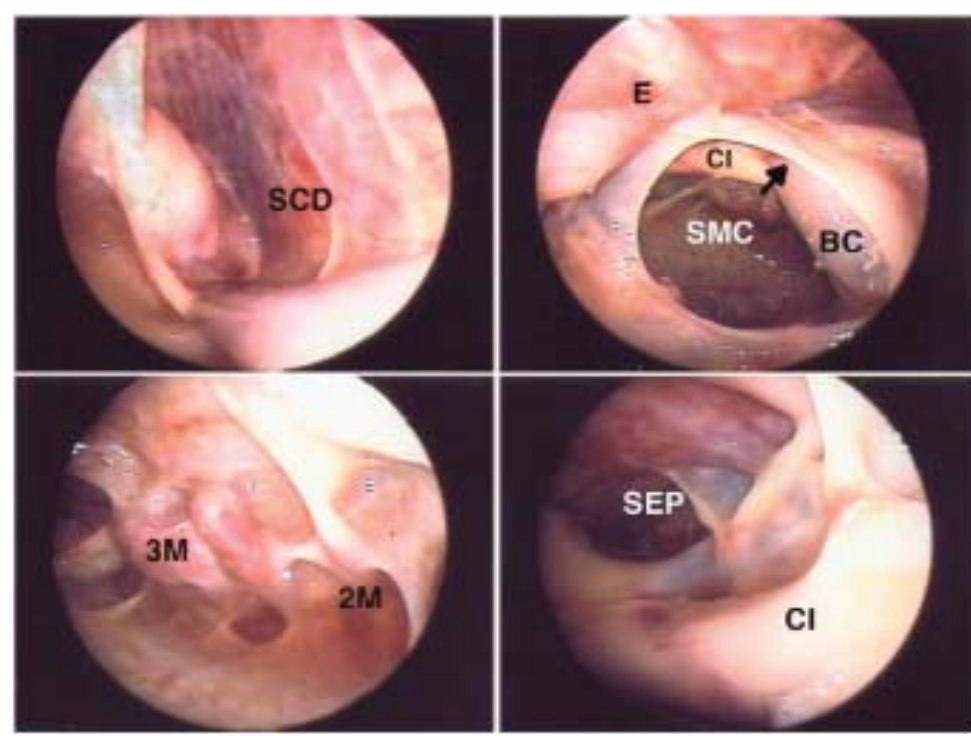

Figura 3 - Imagem obtida a partir do portal SF5 utilizando endoscópio rígido. SCDseio conchal dorsal; E - etmoide; seta - abertura frontomaxilar; CI - canal infraorbitário; BC - bulha conchal; SMC - seio maxilar caudal; 2M ápice raiz do $2^{\circ}$ dente molar; $3 \mathrm{M}$ - raiz do $3^{\circ}$ - dente molar; SEP - seio esfenopalatino.

sugeriram que a criação do portal no seio maxilar rostral fosse guiada por exame radiográfico em animais com idade inferior a cinco anos. PERKINS et al. (2009a) avaliaram em 40 peças anatômicas de equinos o acesso aos seios maxilar rostral e conchal ventral por fenestração da bulha conchal ventral através do portal localizado sobre o seio frontal. Segundo os autores, além de extinguir o risco de lesão à raiz do $1^{\circ}$ dente molar, esse acesso proporcionou exame superior dessas cavidades sinusais.

Ciência Rural, v.43, n.12, dez, 2013. 
Adicionalmente, esse acesso permite comunicação direta entre os compartimentos sinusais, que, por sua vez, aumenta a eficácia da lavagem de todos os seios paranasais por um único portal, prevenindo assim o acúmulo de material purulento em um compartimento sinusal individualizado (PERKINS et al., 2009b).

A diminuição gradativa do tamanho das raízes dentárias foi o que mais diferiu e chamou a atenção nos grupos com diferentes idades avaliados. Este fato apresentou maior relevância durante o exame do seio maxilar rostral, onde a qualidade do procedimento foi comprometida nas peças do GI e GII, indo de encontro com o relato de PERKINS et al. (2009a).

Triangulação: a técnica de triangulação possibilita a realização de biópsia, aspiração e até mesmo pequenos procedimentos cirúrgicos. Para sua realização, torna-se necessária a criação de portal adicional, destinado à passagem de instrumental cirúrgico específico. Nesse estudo, três portais para triangulação foram determinados, sendo dois localizados sobre o seio frontal e um sobre o seio maxilar caudal. Devido ao pequeno espaço interno do seio maxilar rostral, o ponto para triangulação não foi determinado neste seio.

Os locais escolhidos foram os que ofereceram menor complexidade na manipulação das estruturas sinusais e que abordaram um maior número delas com o emprego de um único portal. Os locais que atenderam a essas exigências no seio frontal localizavam-se $2,0 \mathrm{~cm}$ rostral e $2,0 \mathrm{~cm}$ caudal ao portal SF5. Nas 20 peças anatômicas utilizadas, o portal 2,0cm rostral ao SF5 foi superior para manipulação do seio conchal dorsal, e o portal caudal ao SF5 para o exame das estruturas localizadas caudalmente. Para a manipulação do etmoide, tanto o portal caudal como o cranial foram satisfatórios.

Na maioria das peças anatômicas avaliadas ( $n=13)$, o portal $2,0 \mathrm{~cm}$ rostral ao portal SF5 conferiu fácil acesso às estruturas do seio maxilar caudal, podendo ser útil nos casos clínicos de afecções, com acometimento tanto do seio frontal como o maxilar caudal. Em três peças, o exame do seio maxilar caudal foi equivalente pelos dois portais. As estruturas acessadas com maior facilidade pelo portal $2,0 \mathrm{~cm}$ cranial ao portal SF5, contidas no seio maxilar caudal, foram: ápice das raízes dentárias, canal infraorbitário, bulha conchal ventral e septo maxilar.

A técnica de triangulação utilizada para o seio maxilar caudal mostrou-se efetiva para acesso das raízes dentárias, septo, canal infraorbitário e bulha conchal ventral. Entretanto, sua utilização é recomendada quando a afecção está localizada somente no seio maxilar caudal. Quando há a suspeita de acometimento tanto do seio frontal como do maxilar caudal, a sinoscopia associada à triangulação deve ser realizada pelo seio frontal, que permite exploração de ambas as cavidades sinusais, como citado por SILVA et al. (2009) para remoção de cisto sinusal.

O portal para triangulação no seio maxilar caudal localizou-se $2,5 \mathrm{~cm}$ rostral ao portal SMC. Em três peças, houve perfuração do septo, sendo necessário novo portal $1,5 \mathrm{~cm}$ rostral ao portal SMC para o desenvolvimento da técnica de triangulação. Segundo RUGGLES et al. (1991) a localização do septo antes do início do procedimento através de exames radiográficos do local evitam tal complicação.

\section{CONCLUSÃO}

A partir dos resultados obtidos, foi possível concluir que os portais de triangulação estipulados permitiram bom acesso do instrumental cirúrgico às estruturas sinusais, com possibilidade de realização de procedimentos cirúrgicos minimamente invasivos, agregando todos os benefícios da sinoscopia relativos ao período de internação, custo e retorno precoce à atividade atlética ou reprodutiva. Embora ambos os endoscópios tenham possibilitado a visualização de todas as estruturas sinusais, a qualidade da imagem foi superior com o endoscópio rígido, permitindo maior riqueza nos detalhes.

\section{COMITÊ DE ÉTICA E BIOSSEGURANÇA}

Apoio Finaceiro: Fundação de Amparo à Pesquisa do Estado de São Paulo (FAPESP). Processo Comitê Bioética: CEUAVET/fmvz-usp 2792

\section{REFERÊNCIAS}

BEARD, W. L.; HARDY, J. Diagnosis of conditions of the paranasal sinuses in the horse. Equine Veterinary Education, v.13, n.5, p.265-273, 2001. Disponível em: <http://onlinelibrary.wiley.com/ doi/10.1111/j.2042-3292.2001.tb00106.x/abstract>. Acesso em: 25 ago. 2013. doi: 10.1111/j.2042-3292.2001.tb00106.x.

DIXON, P. M.; O’LEARY, J. M. A review of equine paranasal sinusitis: medical and surgical treatments. Equine Veterinary Education, v.24, n.3, p.143-158, 2012. Disponível em: <http:// onlinelibrary.wiley.com/doi/10.1111/j.2042-3292.2011.00245.x/ abstract>. Acesso em: 25 ago. 2013. doi: 10.1111/j.20423292.2011.00245.x.

DIXON, P. M. et al. Equine paranasal sinus disease: along-term study of 200 cases (1997-2009): ancillary diagnostic findings and involvement of the various sinus compartments. Equine Veterinary Journal, v.44, p.267-271, 2012a. Disponível em: <http:// onlinelibrary.wiley.com/doi/10.1111/j.2042-3306.2011.00420.x/ abstract>. Acesso em: 25 ago. 2013. doi: 10.1111/j.20423306.2011.00420.x. 
DIXON, P.M. et al. Equine paranasal sinus disease: A longterm study of 200 cases (1997-2009): Treatments and long-term results. Equine Veterinary Journal, v.44, p.272-276, 2012b. Disponível em: <http://onlinelibrary.wiley.com/doi/10.1111/j.2042 3306.2011.00427.x/abstract $>$. Acesso em: 25 ago. 2013. doi: 10.1111/j.2042-3306.2011.00427.x.

FREEMAN, D. E. Paranasal sinuses. In: BEECH, J. Equine respiratory disorders. London: Lea \& Febiger, 1991. p.275-303.

HILLMANN, D. J. Cabeça óssea. In: GETTY, R. Sisson/ Grossman anatomia dos animais domésticos. 5.ed. Rio de Janeiro: Guanabara Koogan, 1975.p. 294-323.

MARQUES, M.S. et al. Diagnóstico e tratamento de hematoma etmoidal por trepanação e sinoscopia frontal em um equino. Veterinária Notícias, v.7, n.2, p.135-138, 2001.

O’LEARY, J. M.; DIXON, P. M. A review of equine paranasal sinusites. Aetiopathogenesis, clinical signs and ancilliary diagnostic techniques. Equine Veterinary Education, v.23, n.3, p.148-159, 2011. Disponível em: <http://onlinelibrary.wiley.com/ doi/10.1111/j.2042-3292.2010.00176.x/abstract>. Acesso em: 25 ago. 2013. doi: 10.1111/j.2042-3292.2010.00176.x.

PERKINS, J. D. et al. Comparison of sinoscopic techniques for examining the rostral maxillary and ventral conchal sinuses of horses. Veterinary Surgery, v.38, p.607-612, 2009a. Disponível em: <http:// onlinelibrary.wiley.com/doi/10.1111/j.1532-950X.2009.00555.X/ abstract>. Acesso em: 25 ago. 2013. doi: 10.1111/j.1532950X.2009.00555.X.

PERKINS, J. D. et al. Sinoscopic treatment of rostral maxillary and ventral conchal sinusitis in 60 horses.Veterinary Surgery, v.38, p.613-617, 2009b. Disponível em: <http://onlinelibrary.wiley.com/ doi/10.1111/j.1532-950X.2009.00556.x/abstract>. Acesso em: 25 ago. 2013. doi: 10.1111/j.1532-950X.2009.00556.x.
PROBST, A. et al. Communications of normal nasal and paranasal cavities in computed tomography of horses. Veterinary Radiology \& Ultrasound, v.46, n.1, p.44-48, 2005. Disponível em: <http:// onlinelibrary.wiley.com/doi/10.1111/j.1740-8261.2005.00008.x/ abstract>. Acesso em: 25 ago. 2013. doi: 10.1111/j.17408261.2005.00008.x.

RUGGLES, A. et al. Endoscopic examination of normal paranasal sinuses in horse. Veterinary Surgery, v.20, n.6,p.418-423, 1991. Disponível em: <http://onlinelibrary.wiley.com/doi/10.1111/j.1532950X.1991.tb00350.x/abstract>. Acesso em: 25 ago. 2013. doi: 10.1111/j.1532-950X.1991.tb00350.x

RUGGLES, A.J . et al. Endoscopic examination and treatment of paranasal sinus disease in 16 horses. Veterinary Surgery, v.22, n.6, p.508-514, 1993. Disponível em: <http://onlinelibrary.wiley. com/doi/10.1111/j.1532-950X.1993.tb00429.x/abstract>. Acesso em: 25 ago. 2013. doi: 10.1111/j.1532-950X.1993.tb00429.x.

SILVA, L.C.L.C. et al. Excisão de hematoma paranasal em equino através de sinoscopia vídeo-assistida. Brazilian Journal of Veterinary Research and Animal Science, v.41, supl. p.240-241, 2004.

SILVA, L.C.L.C. et al. Bilateral sinus cysts in a filly treated by endoscopic sinus surgery. Canadian Veterinary Journal, v.50, p.417-420, 2009. Disponível em: <http://www.ncbi.nlm.nih.gov/ pmc/articles/PMC2657528/pdf/cvj_04_417.pdf>. Acesso em: 25 ago. 2013.

WILLARD, M. D.; SCHULZ, K. Princípios da cirurgia minimamente invasiva. In: FOSSUM, T.W. Cirurgia de pequenos animais. 3.ed. Rio de Janeiro: Elsevier, 2008.p.146.

WOSTER, A. A.; HACKETT, R. P. Equine sinus endoscopy using a flexible endoscope: Diagnosis and treatment of sinus disease in the standing sedated horse. AAEP Procedings, v.45, p.128-130, 1999. 